\title{
COMMON RASHES
}

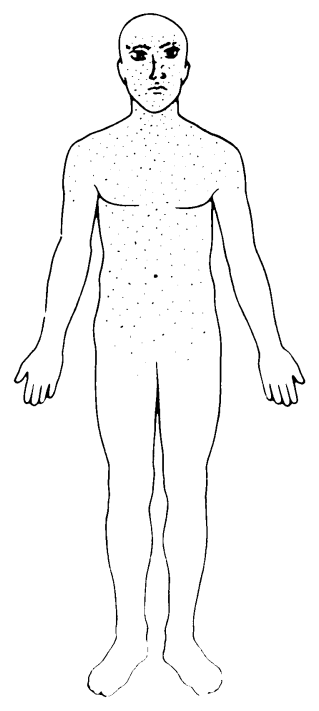

Rashes usually occur as part of one of the common infectious diseases of childhood, but these must be distinguished from dermatological causes. The characteristics of the lesions, distribution, changes with time, and accompanying features help to determine the diagnosis. A previous attack of an infectious disease makes a further attack unlikely, but a high incidence of wrong diagnoses of some rashes, particularly the misdiagnosis of rashes as being caused by rubella, detracts from the value of this history.

\section{Purpura}

\section{Macules and papules}

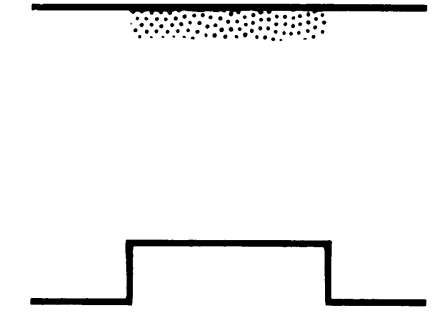

Purpuric lesions are caused by haemorrhages in the skin and they do not disappear on pressure. The child with purpura usually needs to be admitted immediately as he may have meningococcal septicaemia, leukaemia, or idiopathic thrombocytopenic purpura. Henoch-Schönlein purpura is more common than these conditions, however, and is distributed over the extensor surfaces of the limbs as well as the buttocks, and the purpuric lesions are usually raised. If the rash has all the characteristic features admission is not needed.

Macules are discrete lesions that change the colour of the skin, though they fade on pressure. They may be of any size or shape and may be pink or red.

Discrete pink minute macules occur in rubella, when they are accompanied by suboccipital lymphadenopathy. In both roseola and rubella suboccipital lymphadenopathy is pronounced, but in roseola the appearance of the rash coincides with the disappearance of all other symptoms. The child with rubella may have slight fever. In contrast infants with roseola have high fever and irritability for three or four days before the macular rash appears. Despite the high fever the child may play normally.

Papules are solid palpable projections above the surface of the skin. Insect bites are one cause of these lesions, which are called papular urticaria. 


\section{Maculopapular rash}

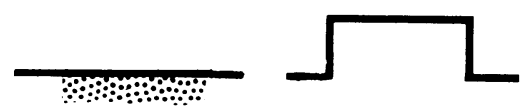

\section{Vesicles}

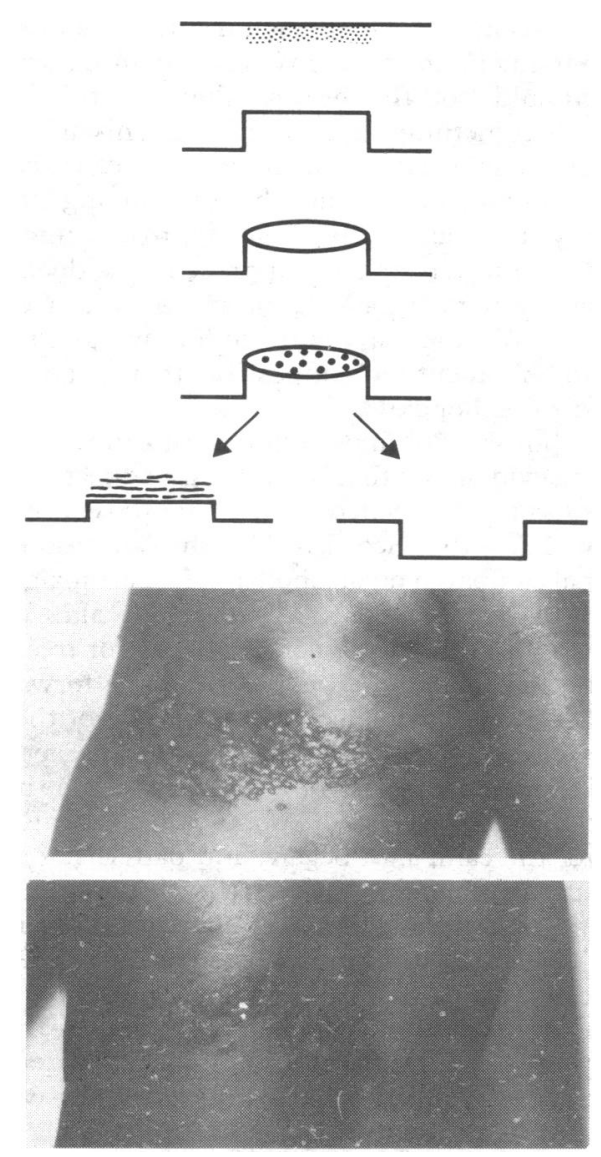

\section{Desquamation}

Vesicles are blisters caused by fluid raising the horny layer of the epidermis. In chickenpox the lesions pass through the stages of macule, papule, and vesicle within 24 hours and crops of rash occur. A fairly clear vesicle on the skin is the diagnostic lesion. In herpes zoster a rash occurs in the distribution of a sensory nerve. Itching may occur before the rash appears.

An infant's first contact with herpes simplex virus may cause vesicles and severe ulcers in the mouth, vulva, or conjunctiva.

Summer outbreaks of hand, foot, and mouth disease are recognised by the pearly white vesicles at these sites due to a Coxsackie virus.

Wheals are raised lesions with a pale centre surrounded by a red area. The rash is accompanied by itching, and the cause is usually not discovered. The rash may be difficult to diagnose in the resolving phase.

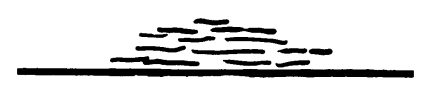

Desquamation is a loss of epidermal cells which produces a "scaly" eruption. Desquamation is found in infantile eczema after the age of 3 years, when it usually affects the flexures, face, and neck but may be more widespread. Psoriasis produces round scaly lesions $0 \cdot 5-1 \mathrm{~cm}$ in diameter on the face, trunk, and limbs.

Dr H B Valman, MD, FRCP, is consultant paediatrician, Northwick Park Hospital and Clinical Research Centre, Harrow.

A maculopapular rash is a mixture of the two types of lesion described above, which tend to be confluent. A maculopapular rash is the typical rash of measles, when it is always accompanied by cough and sometimes from a drug rash, which may itch. Koplik spots have usually disappeared by this stage, but if they persist they may be helpful. The drug may have been given for an upper respiratory tract infection and the clinical picture who have received ampicillin or one of its derivatives. Patients who have of treatment. The rash usually occurs about the 10th day after starting the drug. Usually a second exposure after an interval is needed for a drug rash to occur. 